\title{
Investigation of InAsSbP quantum dot mid-infrared sensors
}

\author{
V. G. Harutyunyan ${ }^{1}$, K. M. Gambaryan ${ }^{1}$, V. M. Aroutiounian ${ }^{1}$, and I. G. Harutyunyan ${ }^{2}$ \\ ${ }^{1}$ Department of Physics of Semiconductors and Microelectronics, Yerevan State University, 1 A. Manoogian, \\ Yerevan 0025, Armenia \\ ${ }^{2}$ Department of Optics, Yerevan State University, 1 A. Manoogian, Yerevan 0025, Armenia \\ Correspondence to: V. G. Harutyunyan (harutyunyan@ysu.am)
}

Received: 13 May 2015 - Accepted: 23 June 2015 - Published: 02 July 2015

\begin{abstract}
This work presents the results of investigations of a low bias mid-infrared(IR) photoconductive cell (PCC) with InAsSbP quantum dots (QDs). The self-assembled nanostructures were grown on an InAs(100) substrate by modified liquid phase epitaxy. The coarsening of the QDs due to Ostwald ripening was discussed. Hysteresis with a remnant capacitance of $0.483 \mathrm{pF}$ and contra-directional oscillations on the PCC's capacitancevoltage characteristic at $78 \mathrm{~K}$ were observed. Additionally, peaks at $3.48,3.68$ and $3.89 \mu \mathrm{m}$ on the room temperature photoresponse spectrum of a quantum dot photoconductive cell were detected. Room temperature photosensing properties were investigated under an irradiation of $3.39 \mu \mathrm{m}$ as well. At a power density of $0.07 \mathrm{~W} \mathrm{~cm}^{-2}$, the surface resistance of quantum dot PCC was reduced by up to $7 \%$. A current responsivity of $0.2 \mathrm{~mA} \mathrm{~W}^{-1}$ was measured at an applied voltage of $8 \mathrm{mV}$.
\end{abstract}

\section{Introduction}

The importance of the mid-infrared(IR) range is attributed to the transmission window of the atmosphere, absorption spectra of some industrial gases, etc. To satisfy the demands of state-of-the-art infrared photodetectors, nowadays quantum well infrared photodetectors (QWIPs) and quantum dot infrared photodetectors (QDIPs) are of great interest. The QWIP approach has the advantages of availability of a mature III-V fabrication technology and multi-spectral capability. This type of photodetector has a narrow absorption spectrum that can be tuned by varying the quantum well width and barrier layer compositions. There is a large range of material combinations available allowing the tailoring of band gaps through stacking of thin layers. As for QDIPs, this type of photodetector is predicted to have superior performances compared to QWIPs (Phillips, 2002), such as sensitivity to normal incidence infrared radiation, low dark current, and high responsivity and detectivity. Many semiconductor material systems, such as InGaAs/GaAs, InAsSb/GaAs and InAs/GaAs, were investigated for QDIPs (Bhattacharya et al., 2005; He et al., 2010 and Lao et al., 2013). HgCdTe (MCT) is a well-established solid solution, which has been the dominant system for mid- and long-infrared photodetectors. However, MCT suffers from instability and nonuniformity problems over a large area due to high $\mathrm{Hg}$ vapour pressure. Theoretical studies predicted that only type-II superlattice photodiodes and QDIPs are expected to compete with $\mathrm{HgCdTe}$ photodiodes (Martyniuk and Rogalski, 2008). The InAs-InSb-InP system could be discussed as an alternative material system for mid-IR applications. InAsSbP quaternary alloy was successfully used for nucleation of quantum dots (QDs). In the first experiment to grow nanostructures with this quaternary composition, GaAs was used as a substrate (Krier, 1999). In that work the grown structure was characterized by photoluminescence (PL) measurements at $4 \mathrm{~K}$ temperature and the PL peak at $1.65 \mu \mathrm{m}$ was observed. Recently, InAsSbP QDs were successfully grown on InAs substrates as well (Gambaryan, 2010, and Gambaryan et al., 2012). It was shown that using InAs substrates leads to the sensitivity in the mid-IR range (Gambaryan et al., 2011, and Harutyunyan, 2013). Among quantum-size object fabrication techniques, the self-organized Stranski-Krastanow (S$\mathrm{K})$ growth mode is an important one by which dislocationfree nanostructures can be produced (Stranski and Krastanov, 
1938). Depending on the growth conditions, the elastic strain can be relaxed by the formation of QDs, quantum rings or even unique island-pit pairs (Gambaryan, 2010 and Gambaryan et al., 2012). In the S-K method, the last stage of nucleation in solid and liquid solutions is often characterized by the growth of the largest (supercritical) clusters and solving of small (subcritical) ones, which results in the decrease in the density of clusters with the increase in their sizes. Such a process of coarsening of clusters is usually termed "Ostwald ripening" in the literature, in connection with his studies performed at the beginning of the twentieth century (Ostwald, 1901). A major advance in the theory of Ostwald ripening was made by Lifshitz and Slyozov (1961) and followed by Wagner (1961).

In this paper, we report our efforts to grow InAsSbP QDs on InAs substrates by modified liquid phase epitaxy. Grown nanostructures were studied by atomic force microscope (AFM) and scanning electron microscope (SEM). Quantum dot mid-IR photodetectors were fabricated in the form of photoconductive cells (PCCs) made of InAs substrates with InAsSbP QDs. Electrophysical and optoelectronic properties of fabricated photodetectors are reported as well.

\section{Experiment}

To grow QDs, a modified liquid phase epitaxy (MLPE) was used. For the formation of a liquid phase, In $(7 \mathrm{~N})$ was used as a solvent and InAs, InP and $\mathrm{Sb}(6 \mathrm{~N})$ were used as solutes. A lattice mismatch of $2 \%$ between an $n-\operatorname{InAs}(100)$ substrate with a thickness of $400 \mu \mathrm{m}$ and a wetting layer was chosen to provide the growth of InAsSbP nanostructures in $\mathrm{S}-\mathrm{K}$ growth mode. The surface morphology, size distribution and density of quantum dots were studied using an atomic force microscope (AFM - Asylum Research MFP3D). Additionally, large-scale surfaces of grown structures were investigated using a high-resolution scanning electron microscope (HR-SEM: FEI Nova 600-Dual Beam). Au-Cr evaporation was performed to form contacts on the sample's surface. Actually, a fabricated photoconductive cell (PCC) consists of an InAs substrate, unencapsulated InAsSbP QDs and contacts (Fig. 1). The active area of the photoconductive cell is $0.83 \mathrm{~mm}^{2}$. Capacitance-voltage $(\mathrm{C}-\mathrm{V})$ characteristics were investigated by high-precision capacitance spectrometry (QuadTech-1920 precision LCR meter). Mid-IR photoresponse spectra were measured by an IRS-21 spectrometer. Optoelectronic properties were investigated using a $\mathrm{He}-\mathrm{Ne}$ laser at an irradiation wavelength of $3.39 \mu \mathrm{m}$.

\section{Results and discussion}

Figure 2 presents the AFM images of unencapsulated InAsSbP QDs nucleated from the quaternary In-As-Sb$\mathrm{P}$ liquid phase during $10 \mathrm{~min}$ at a growth temperature of $550^{\circ} \mathrm{C}$. From Fig. 2a one can notice that QDs are sufficiently

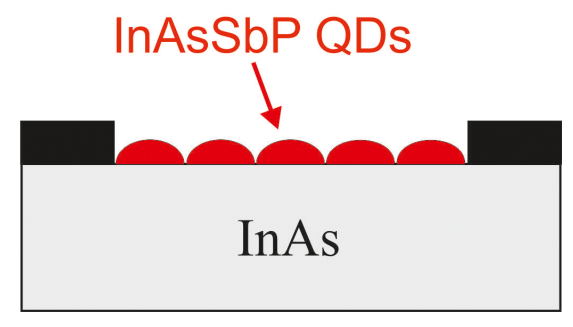

Figure 1. Schematic of the fabricated photoconductive cell with QDs.

uniformly distributed over an $S=4.5 \mu \mathrm{m} \times 5 \mu \mathrm{m}$ substrate surface. The average surface density and heights of grown QDs were found to be $7 \times 10^{9} \mathrm{~cm}^{-2}$ and $21 \mathrm{~nm}$, respectively. The average aspect ratio between QDs' widths and heights equals $2.5 \pm 0.5$. The SEM and AFM images of the nanostructures nucleated at $20 \mathrm{~min}$ are shown in Fig. 3. The enlarged views of regions denoted in Fig. 3a by white and black cycles are presented as insets in the same image. One can notice the process of Ostwald ripening and bimodal growth of QDs. First, an array of small-size QDs distributed over a relatively large area $\left(\sim 300 \mu \mathrm{m}^{2}\right)$ can be seen and, second, large-diameter QDs with much lower surface density are quite visible as well. We believe that coarsening of large QDs occurs via solving of small QDs with subsequent surface diffusion of dissolved material towards large QDs. The detailed analyses of measurements showed that small-size QDs have a mainly spherical shape, while large ones with a diameter of $\sim 50 \mu \mathrm{m}$ and larger are ellipsoidal. Increasing the growth time leads to the transformation of QDs' distribution functions versus their sizes. In particular, distribution of QDs grown at 10 min was well fitted by the GramCharlier function, while for 20 and $30 \mathrm{~min}$ by the Gaussian and Lifshits-Slezov functions, respectively (Aroutiounian et al., 2013). A similar change in the distribution function was observed at nucleation of silicon nano-islands on a $\mathrm{Si}(001)$ surface (Bartelt et al., 1996). It was shown, by means of detailed analysis of individual islands and close-lying regions of substrate, that merging and coarsening of QDs occurs via dissolving of small QDs with subsequent surface diffusion of dissolved substances towards larger QDs, leading to their further coarsening. We assume that a similar mechanism of QD coarsening occurs in our case as well. Our studies showed also that at further increase of growth time ellipsoidal QDs are transformed to elongated islands with dimensions up to a few micrometres. For the fabrication of the photodetector, the sample prepared at $10 \mathrm{~min}$ growth time was used. Figure 4 shows the dark current-voltage (I-V) characteristic of the PCC. Nonlinear I-V behaviour is found at around $\sim 60 \mathrm{mV}$ voltage. The result of the $\mathrm{C}-\mathrm{V}$ characterization performed at $78 \mathrm{~K}$ and $10^{6} \mathrm{~Hz}$ frequency is presented in Fig. 5. Measurements were performed by increasing the signal voltage from 0 up to $0.9 \mathrm{~V}$ with further decreasing back to $0 \mathrm{~V}$ as denoted by arrows in the figure. In addition, it can be seen that oscil- 


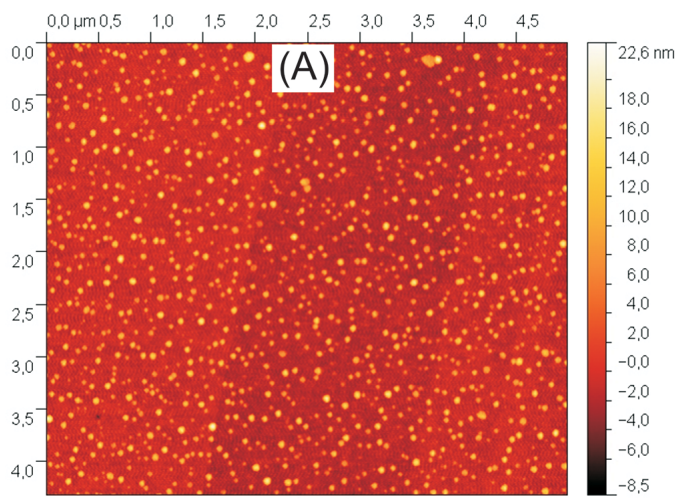

(B)

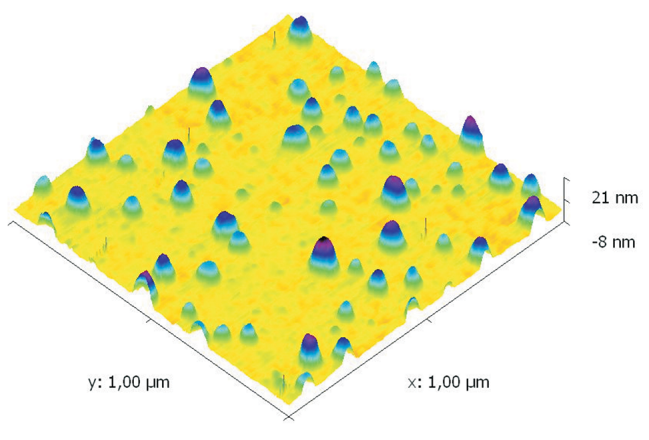

Figure 2. AFM images of InAsSbP QDs grown on an InAs substrate. (a) - plane view; (b) - oblique view.

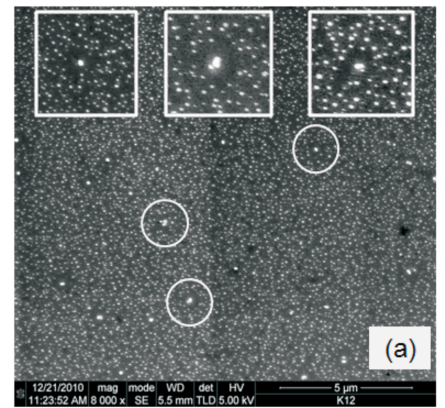

(b)

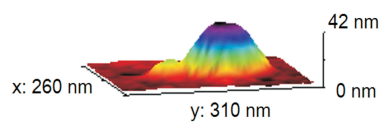

(c)

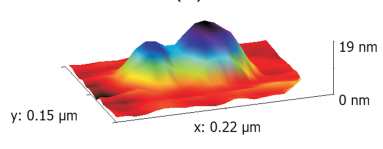

Figure 3. (a) - SEM image of InAsSbP QDs grown by LPE on an InAs substrate $\left(S=17 \times 17 \mu \mathrm{m}^{2}\right)$; (b), (c) - AFM images of QD coalescence.

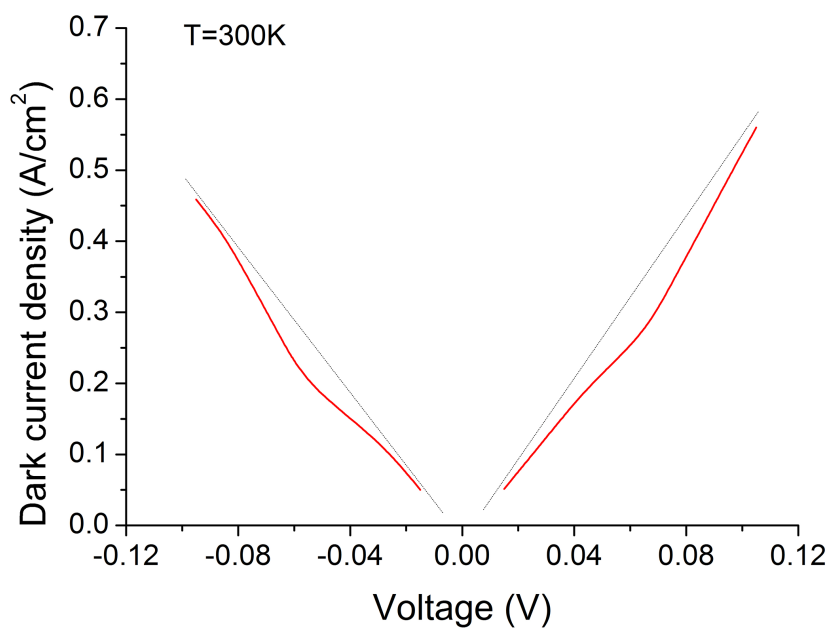

Figure 4. PCC's dark current density versus applied voltage.

lations on the $\mathrm{C}-\mathrm{V}$ curve are observed. Oscillations observed during increasing voltage are opposite-directed to the oscillations observed during further decreasing of the voltage. From Fig. 5 it can also be seen that the capacitance hysteresis with a remnant capacitance of $\Delta \mathrm{C}=0.483 \mathrm{pF}$ is revealed. We as-

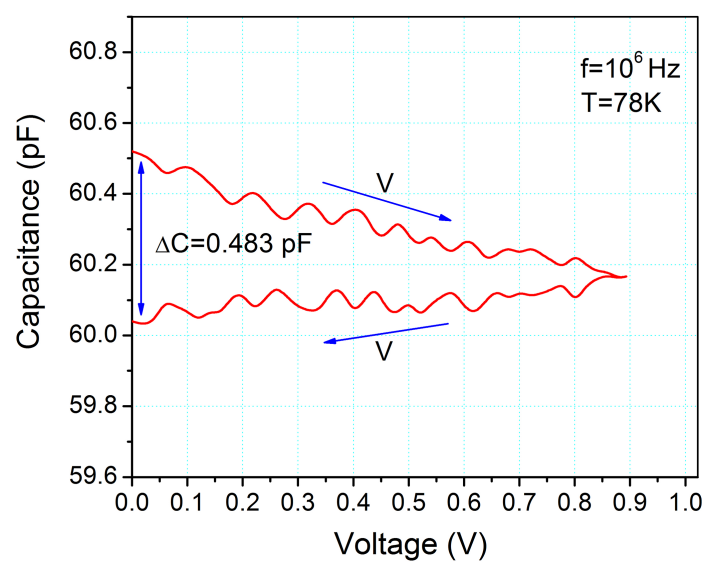

Figure 5. Capacitance-voltage characteristic of the PCC.

sume that opposite-directed oscillations revealed on the $\mathrm{C}-\mathrm{V}$ curve occur due to the structure's total charge oscillations, which are caused by the depletion and occupation of the QDs' energy levels. Detected hysteresis can be explained by the remnant polarization in the structure due to spatial separation of the charge carriers in type-II InAsSbP QDs (Gambaryan et al., 2011) which are conserved after shutting off the voltage. For photoresponse measurements, a testing infrared photodetector (TIP) was fabricated and used. The TIP is a traditional photoconductive cell made of InAs bulk crystal (without QDs). The photoresponse spectra of the samples at room temperature were investigated by applying a $2 \mathrm{mV}$ low bias. For TIP only one peak at $3.48 \mu \mathrm{m}$ corresponding to the energy band gap of InAs $(\mathrm{Eg}=0.355 \mathrm{eV})$ was observed (Fig. 6). It can obviously be seen that the PCC, in comparison with the TIP, has a photoresponse spectrum extended to longer wavelengths up to $4 \mu \mathrm{m}$ with a narrow peak at $3.48 \mu \mathrm{m}$. As was shown in our previous works, InAsSbP QDs grown on InAs form type-II band alignment (Gambaryan, 2010). The schematic of the band structure of the grown structure used for PCC in this work is presented in 


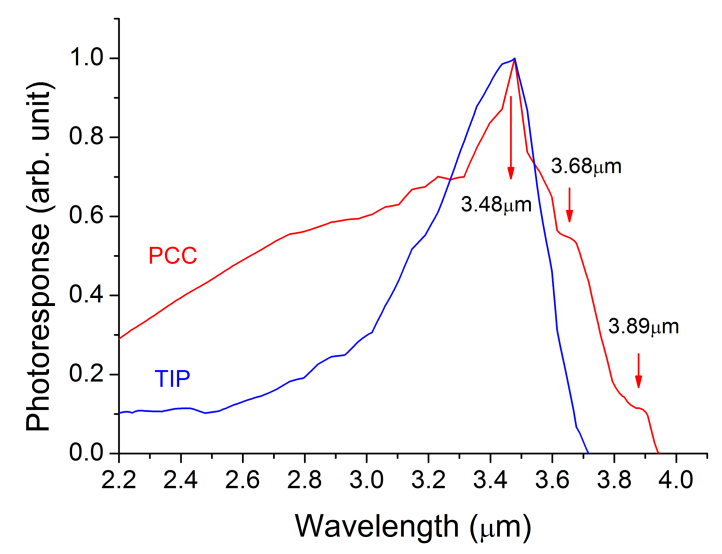

Figure 6. Photoresponse spectra of the TIP and PCC at room temperature.

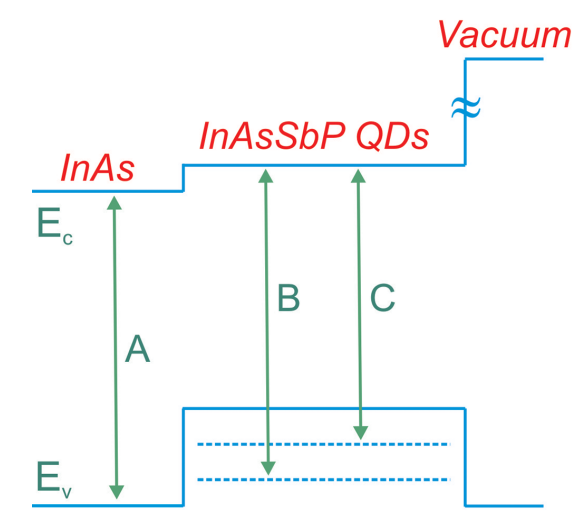

Figure 7. Schematic of the band structure of the type-II InAsSbP QDs grown on InAs.

Fig. 7. Photoresponse peaks at $3.68 \mu \mathrm{m}(B=0.337 \mathrm{eV})$ and $3.89 \mu \mathrm{m}(C=0.319 \mathrm{eV})$ wavelengths could be explained by the transitions into sub-band-gap levels created by QDs. The surface resistance of the samples was also investigated under irradiation at $3.39 \mu \mathrm{m}$. The relative surface resistance change in the PCC versus power density is plotted in Fig. 8. At a power density of $0.07 \mathrm{~W} \mathrm{~cm}$, the resistance of the PCC was reduced by $7 \%$. Meanwhile, the surface resistance of the TIP at the same density was reduced by $2 \%$. Thus, due to the growth of InAsSbP QDs, the resistance change sensitivity of the PCC is increased by 3.5 times. The current responsivity of the PCC was investigated at power density of $0.05 \mathrm{~W} \mathrm{~cm}^{-2}$ (Fig. 9). The current responsivity of $0.2 \mathrm{~mA} \mathrm{~W}^{-1}$ was achieved at an operating voltage of $8 \mathrm{mV}$, which exceeds the responsivity of the TIP by 20 times at the same power density and bias. As a result, fabricated photoconductive cells with InAsSbP quantum dots are important for low bias operation at the mid-IR range. Reported results could be important for developing the idea of photoconductive cells with quantum dots for next-generation infrared devices.

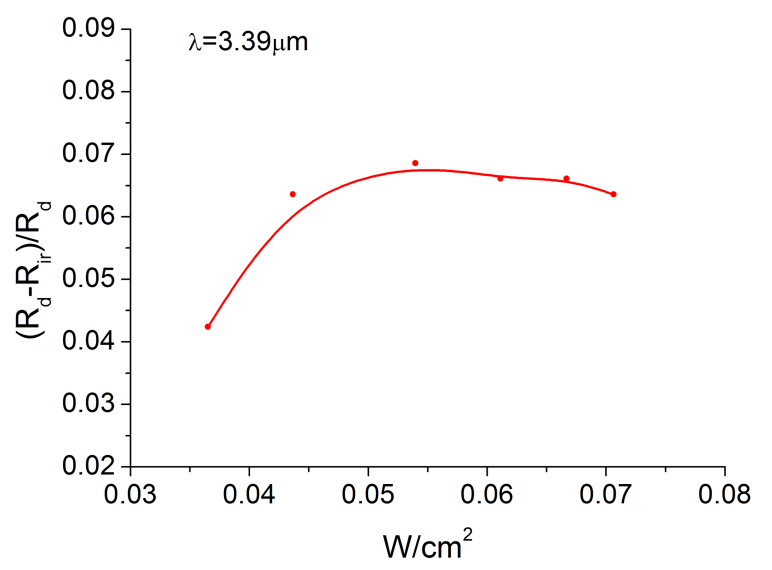

Figure 8. Relative resistance change dependence on power density. $R_{\mathrm{d}}$ is the dark resistance and $R_{\mathrm{ir}}$ is the resistance under irradiation.

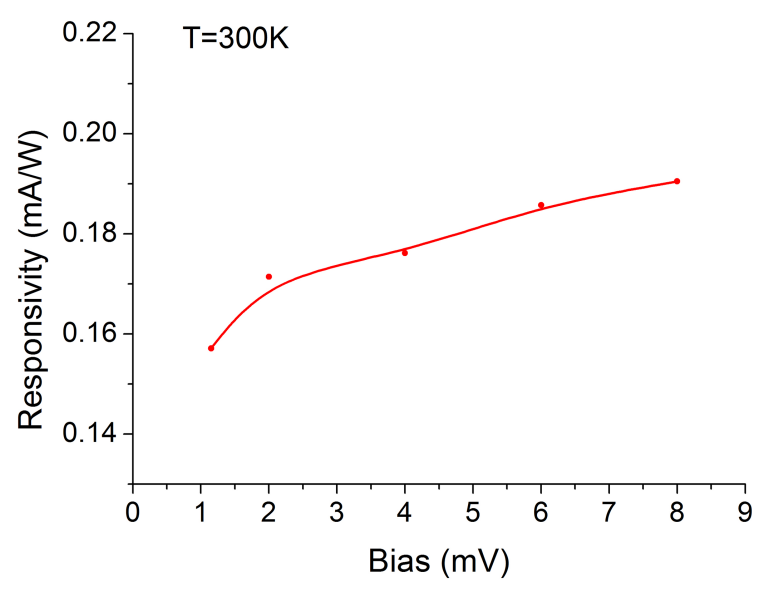

Figure 9. PCC's current responsivity versus bias.

\section{Conclusions}

A low bias mid-IR photoconductive cell with InAsSbP QDs was fabricated. Nonlinear current-voltage behaviour was found at room temperature. The Ostwald ripening for InAsSbP nanostructures is studied. Specific oppositedirected oscillations and hysteresis with a remnant capacitance of $\Delta \mathrm{C}=0.483 \mathrm{pF}$ were observed at $78 \mathrm{~K}$ temperature. PCC's room temperature photoresponse spectrum is extended to longer wavelengths up to $4 \mu \mathrm{m}$. Besides red shift, two additional peaks were observed at 3.68 and $3.89 \mu \mathrm{m}$ wavelengths. It was shown that under an irradiation of $3.39 \mu \mathrm{m}$, PCC's surface resistance changes 3.5 times more than that of TIP. Applying a $8 \mathrm{mV}$ bias, a current responsivity of $0.2 \mathrm{~mA} \mathrm{~W}^{-1}$ for PCCs was measured. This value exceeds the TIP's responsivity by 20 times. Thus, fabricated low bias photodetectors with InAsSbP QDs can be successfully used in the mid-IR range. 
Acknowledgements. This work was performed in the framework of basic financial support by the State Committee of Science of Armenia and the NATO SFP 984597 grant.

Edited by: W. A. Minkina

Reviewed by: two anonymous referees

\section{References}

Aroutiounian, V. M., Gambaryan, K. M., Harutyunyan, V. G., Soukiassian, P. G., Boeck, T., Schmidtbauer, J., and Bansen, R.: The Ostwald Ripening at Nanoengineering of InAsSbP Spherical and Ellipsoidal Quantum Dots on InAs (100) Surface, J. Contemp. Phys. (Armenian Academy of Sciences), 48, 37-42, 2013.

Bartelt, N. C., Theis, W., and Tromp, R. M.: Ostwald ripening of two-dimensional islands on $\mathrm{Si}(001)$, Phys. Rev. B, 54, 1174111751, 1996.

Bhattacharya, P., Su, X. H., Chakrabarti, S., Ariyawansa, G., and Perera, A. G. U.: Characteristics of a Tunneling Quantum-dot Infrared Photodetector Operating at Room Temperature, Appl. Phys. Lett., 86, 191106-1-1191106-3, 2005.

Gambaryan, K. M.: Nucleation of InAsSbP Quantum, Dots and Pits on InAs(100) Substrate, Nanoscale Res. Lett., 5, 587-591, 2010.

Gambaryan, K. M., Aroutiounian, V. M., and Harutyunyan, V. G.: Photovoltaic and optoelectronic properties of InAs( 100$)$-based photoconductive cells with quantum dots and nanopits, Infrared Phys. Technol., 54, 114-120, 2011.

Gambaryan, K. M., Aroutiounian, V. M., Harutyunyan, V. G., Marquardt, O., and Soukiassian, P. G.: Room temperature magnetoelectric properties of type-II InAsSbP quantum dots and nanorings, Appl. Phys. Lett., 100, 033104-1-033104-4, 2012.
Harutyunyan, V., Gambaryan, K., and Aroutiounian, V.: Novel narrow band-gap InAsSbP-based quantum dot mid-infrared photodetectors: fabrication, optoelectronic and electrophysical properties, J. Nanosci. Nanotechnol., 13, 799-803, 2013.

He, J., Reyner, C. J., Liang, B. L., Nunna, K., Huffaker, D. L., Pavarelli, N., Gradkowski, K., Ochalski, T. J., Huyet, G., Dorogan, V. G., Mazur, Y. I., and Salamo, G. J.: Band alignment tailoring of InAs $\mathrm{As}_{1-x} \mathrm{Sb}_{x} / \mathrm{GaAs}$ quantum dots: Control of type I to type II transition, Nano Lett., 10, 3052-3056, 2010.

Krier, A., Labadi, Z., and Hammiche, A.: InAsSbP quantum dots grown by liquid phase epitaxy, J. Phys. D Appl. Phys., 32, 2587 2589, 1999.

Lao, Y.-F., Wolde, S., Perera, A. G. U., Zhang, Y. H., Wang, T. M., Liu, H. C., Kim, J. O., Schuler-Sandy, T., Tian, Z.-B., and Krishna, S. S.: InAs/GaAs p-type quantum dot infrared photodetector with higher efficiency, Appl. Phys. Lett., 103, 241115-1241115-4, 2013.

Lifshitz, I. M. and Slyozov, V. V.: The kinetics of precipitation from supersaturated solid solutions, J. Phys. Chem. Solids, 19, 35-50, 1961.

Martyniuk, P. and Rogalski, A.: Quantum-dot infrared photodetectors: Status and outlook, Prog. Quant. Electron., 32, 89-120, 2008.

Ostwald, W.: Blocking of Ostwald ripening allowing long-term stabilization, Z. Phys. Chem., 37, 385-390, 1901.

Phillips, J: Evaluation of the fundamental properties of quantum dot infrared detectors, J. Appl. Phys., 91, 4590-4594, 2002.

Stranski, I. N. and Krastanov, L.: Zur theorie der orientierten ausscheidung von ionenkristallen aufeinander, Sitz. Ber., Akad. Wiss., Math.-naturwiss. Kl. Abt. IIb, 146, 797-810, 1938.

Wagner, C.: Theorie der alterung von niderschlagen durch umlösen (Ostwald Reifung), Z. Elektrochemie, 65, 581-591, 1961. 